\title{
Replacing the wild type loxP site in BACs from the public domain with lox66 using a lox66 transposon
}

Pradeep K Chatterjee ${ }^{1,2^{*}}$, Leighcraft A Shakes ${ }^{1,2}$, Naima Stennett ${ }^{2,3}$, Vanessa L Richardson ${ }^{2,3}$, Tennison L Malcolm ${ }^{1,2}$, Ken R Harewood ${ }^{2,3}$

\begin{abstract}
Background: Chromatin adjoining the site of integration of a transgene affects expression and renders comparisons of closely related transgenes, such as those derived from a BAC deletion series retrofitted with enhancer-traps, unreliable. Gene targeting to a pre-determined site on the chromosome is likely to alleviate the problem.

Findings: A general procedure to replace the loxP site located at one end of genomic DNA inserts in BACs with lox66 is described. Truncating insert DNA from the loxP end with a Tn10 transposon carrying a lox66 site simultaneously substitutes the loxP with a lox66 sequence. The replacement occurs with high stringency, and the procedure should be applicable to all BACs in the public domain. Cre recombination of loxP with lox66 or lox71 was found to be as efficient as another loxP site during phage P1 transduction of small plasmids containing those sites. However the end-deletion of insert DNA in BACs using a lox66 transposon occurred at no more than 20\% the efficiency observed with a loxP transposon. Differences in the ability of Cre protein available at different stages of the P1 life cycle to recombine identical versus non-identical lox-sites is likely responsible for this discrepancy. A possible mechanism to explain these findings is discussed.

Conclusions: The loxP/lox66 replacement procedure should allow targeting BACs to a pre-positioned lox71 site in zebrafish chromosomes; a system where homologous recombination-mediated "knock-in" technology is unavailable.
\end{abstract}

\section{Findings}

\section{Research Hypothesis}

Expression of a transgene integrated into the germline of an animal is influenced by i) variation in copy number of transgene and ii) the effect of chromatin adjoining the site of integration [1]. Thus expression comparisons of closely related transgenes, such as those derived from a BAC deletion series retrofitted with enhancer-traps [2], become unreliable. Targeting transgenes to the same site on the chromosome alleviates the problem, and both "knock-in" technology using homologous recombination [1] and insertion of cDNA plasmids into a loxP site have been used in previous studies [3].

\footnotetext{
* Correspondence: pchatterjee@nccu.edu

'Department of Chemistry, North Carolina Central University, 1801 Fayetteville Street, Durham, NC 27707, USA
}

Because the lox-Cre recombination reaction/equilibrium favors excision, mutant sites such as lox66 and lox71 have been constructed to stably incorporate small plasmid DNA into chromosomes of plants and mouse ES cells $[4,5]$. BACs have not been used similarly, presumably because altering the loxP or lox511 sites flanking insert DNA in BACs is challenging. We describe a general procedure that readily overcomes this particular hurdle of converting a loxP to a lox66 in BACs.

\section{Materials and methods}

Two BAC clones from the zebrafish genomic library, $\mathrm{CH} 211-192 \mathrm{O} 20$, \& $\mathrm{CH} 211-43 \mathrm{O} 16$ designated here as BACs C \& D respectively, were purchased from BAC/PAC resources, Oakland, California. These zebrafish BACs are in the pTARBAC2.1 vector. BACs C and D are of size 138.6 and $144.3 \mathrm{~kb}$, respectively, as deduced from the 
location of their ends on the zebrafish chromosome 9 BAC contig of this region. They appear closer to $130 \mathrm{~kb}$ on FIGE. End-deletions of insert DNA in BACs were generated with lox66 transposons using procedures identical to those described earlier for loxP transposons $[6,7]$. Briefly, the lox66 transposon plasmid was introduced into the bacterial host containing the BAC using the calcium chloride transformation procedure. Transposition into BAC DNA was initiated by inducing the cells with IPTG. Cre recombinase was provided by infecting these cells with phage $\mathrm{P} 1$. The resulting end-deleted BACs were packaged as linear DNA in P1 heads, and used to infect fresh bacteria to regenerate the lox66 substituted BAC plasmids. Procedures for DNA isolation/purification from BAC deletions, FIGE analysis, end-sequencing of BAC deletions with transposon-based primers have been described earlier $[8,9]$. Identical procedures were also followed with the BAC deletions generated with lox66 enhancer-trap transposons. Primers used for sequencing the newly created end of BACs are:

\section{Seq 1......5' d GACAAGATGTGTATCCACCTTAAC 3 \\ Seq 4-compliment......5' d CCGTTTTTAT- CAGGCTCTGGGAG 3' \\ LF8-compliment......5' d CTTGATTCCATT- CATCTGTAGTG 3'}

\section{Results}

\section{Cre-recombination of mutant lox sites}

Mutant lox sites generated over the years [4,5,10-15] are of two categories: i) mutations in the $8 \mathrm{bp}$ asymmetric spacer and ii) mutations in the $13 \mathrm{bp}$ inverted repeats (Figure 1). The $8 \mathrm{bp}$ spacer region is thought to form Holiday-like structure with perfect base pairing required during the recombination process. Single nucleotide differences in spacer of two lox sites render recombination between them very inefficient [10-13,16]. Nevertheless varying degrees of promiscuity in recombining different spacer-mutant lox sites have been reported; using both partially purified Cre-extracts in vitro $[10,11,14,15]$, and Cre over-expressed in cells $[12,13,15,17]$. High levels of stringency in vivo can be achieved however with Cre protein expressed from its native source namely, a phage P1 infection $[7,16]$.

Nucleotide substitutions in the $13 \mathrm{bp}$ inverted repeats (palindromic arms) appear not to severely limit its recombination with a wild type loxP sequence, and this tolerance increases for substitutions towards the outer ends of these inverted repeats [18]. Cre protein binds to these $13 \mathrm{bp}$ palindromic arms to recombine two lox sites $[18,19]$, and the protein-protein interactions between Cre molecules appear to compensate for possible distortions in Cre binding to mutant-arm DNA.
Wild-type loxP and the arm-mutant lox66 have identical spacers, and recombine well with Cre [5]. We used this rationale to substitute a lox66 for the wild-type loxP site in BACs while truncating the insert DNA using a lox66 transposon (Figure 1).

\section{Stringency in Cre recombinations between wild type and mutant lox-sites}

While cross recombination between loxP and lox511 had been reported to occur at efficiencies ranging from 5 to $100 \%$ in a variety of settings that expressed Cre constitutively $[11-13,15,17]$, it was determined to be no more than $0.5 \%$ in our BAC end-deletion procedures where Cre protein is generated by a phage P1 infection $[7,16]$. The possibility that phage P1 infection-derived Cre protein in our procedures might prove too stringent to allow efficient recombination between loxP and lox66, despite the fact that lox66 is an arm mutant and not a spacer mutant like lox511, was explored first by testing the ability of phage P1, carrying wild type loxP, to transduce a plasmid containing a lox66 site [16].

Testing Cross Recombination between loxP of phage P1 \& mutant lox sites in plasmids carrying antibiotic resistance genes with phage P1-derived Cre protein

$E$. Coli with small plasmids carrying one of several mutant lox sites and a gene conferring resistance to an antibiotic were each infected with phage P1. The P1 lysates were used to infect fresh bacteria, and plated on LB agar containing the antibiotic whose resistance gene was carried by the plasmid [16]. The results are shown in Table 1.

The results indicate that phage P1 can transduce a lox66 plasmid just as efficiently as a wild type loxP plasmid (compare rows 1 \& 2 with 4 in Table 1). However, phage P1 was unable to transduce plasmids with the spacer mutants lox511, lox5171 and lox2272 (mutants described in references 10 and 11), and these were used as negative controls in the experiment (rows 3, $7 \& 8$ ). The data shown are from two independent experiments. Note that both the lox "arm mutants", lox66 and lox71, are efficiently transduced, while all of the "spacer mutants", lox511, lox2272 and lox5171 are not (compare rows $1,2,5,6$ with $3,7,8$ ). There was a quantitative difference between $l o x 511$ and $l o x 5171$ or lox2272: while the latter two produced zero colonies on both runs, the lox511 produced 2 and 4 colonies in the two experiments. The results are in line with earlier findings $[16,7,5]$, although the high efficiency of transducing lox66 and lox71 plasmids by wild type loxP in phage $\mathrm{P} 1$ under our more stringent lox-Cre recombination conditions was surprising.

Having demonstrated efficient cross recombination between lox66 and loxP using phage P1 derived Cre protein, we tested the lox66 transposons to generate 


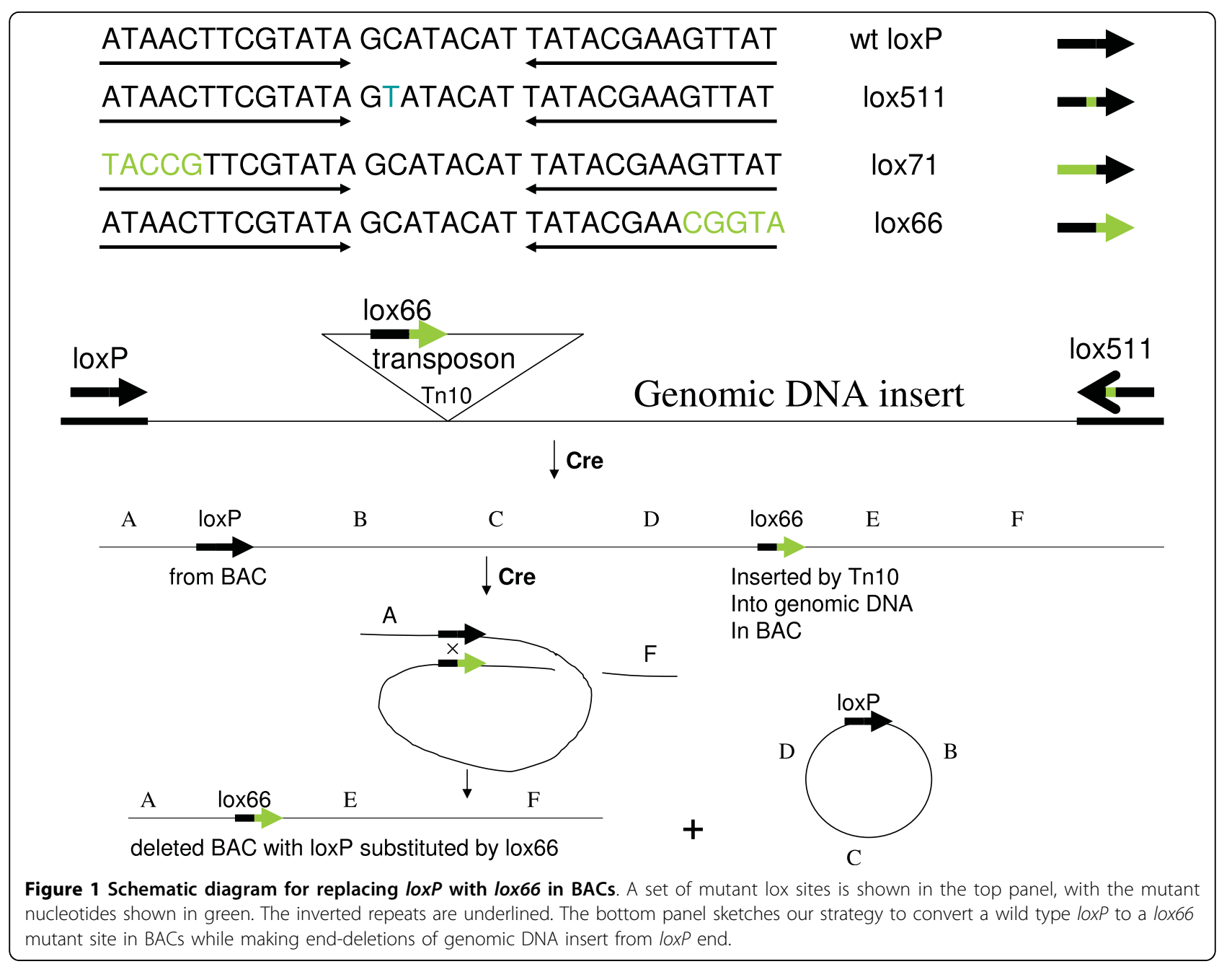

Table 1 Phage P1 transduction of plasmids with mutant lox sites

\begin{tabular}{llll}
\hline Clone & Marker & Lox Site & Transduction with phage P1 \\
\hline a & ampR & lox66 & ++++ \\
b & ampR & lox66 & ++++ \\
c & ampR & lox511 & 0 \\
d & ampR & wt loxP & ++++ \\
g & ampR & lox71 & ++++ \\
h & ampR & lox71 & ++++ \\
i & camR & lox5171 & 0 \\
& & & \\
j & camR & lox2272 & 0 \\
& & & \\
k & kanR & wt loxP & ++++ \\
l & kanR & wt loxP & ++++ \\
\hline $0-2$ colonies/plate: 0 & & \\
$500-1000$ colonies/plate: & ++++ &
\end{tabular}

end-deletions of insert DNA in BACs. The following lox66 markerless transposon plasmids, similar to our other markerless transposons reported earlier [20,7], were constructed (Figure 2).

\section{Progressive end-deletions of insert DNA in BACs with pTnLox66(B)markerless and pTnLox66(B)markerless Enhancer-Trap transposons}

End deletions of genomic DNA in BAC clone $\mathrm{C}$ was made with pTnLox66(B)markerless transposon (Figure 2 , top panel) exactly as described earlier with loxP markerless transposons [20,7,2]. DNA isolated from a set of deletions is shown in lanes 3-11, Figure 3A. Note that inversions of BAC DNA resulting from transpositions of lox66 in the opposite orientation to that in the BAC vector (schematic in Figure $3 \mathrm{~B}$ ) are not recovered because the starting BAC clones are larger than P1headful length [20].

End-deletions of insert DNA in a different BAC clone D with pTnLox66(B)markerless Enhancer-Trap 


\section{Lox66 Transposon plasmids}

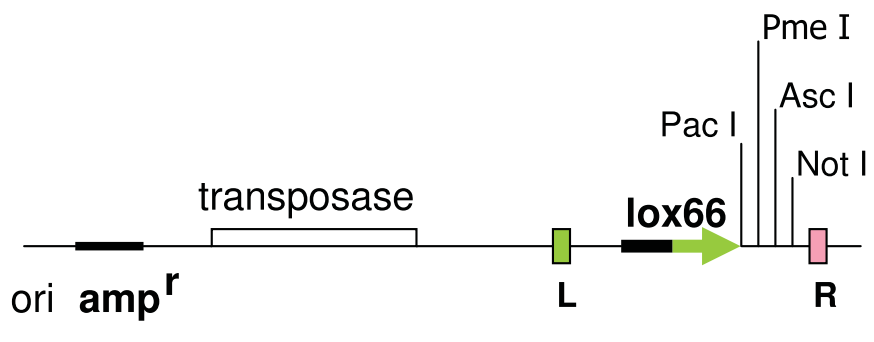

pTnLox66(B)markerless

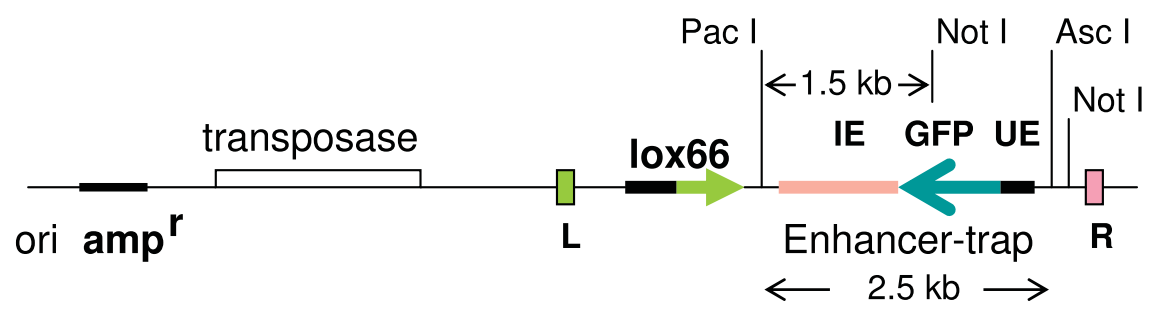

\section{pTnLox66(B)markerless Enhancer-Trap}

Figure 2 Schematic representation of the two lox66 transposons constructed. Both use the same framework of markerless transposons with loxP or lox511 [20,7]. The enhancer-trap is adapted from [2].

transposon (schematic in lower panel of Figure 2) is displayed in lanes 7-11 of Figure 3, panel A. Not I sites in pTARBAC2.1 vector DNA in BAC clones is shown in panel $B$. Note that the 3827 bp of DNA between $\operatorname{lox} P$ and the Not I site at 2779 is replaced during recombination of transposed lox66 and loxP in $\mathrm{BAC}$ vector. Thus in deletions with the pTnLox66(B) markerless transposon the BAC vector band obtained with Not I digestion is $\sim 6.6 \mathrm{~kb}$ (see lanes $3-5$ of Figure 3A). Deletions with pTnLox66(B)markerless EnhancerTrap transposon results in a Not I vector DNA band larger by $1.5 \mathrm{~kb}$, (lanes 7-11 Figure 3A), due to the Not I site being further away in the enhancer-trap (see lower panel of Figure 2). BAC insert DNA sequencing with the transposon based primer Seq 1 [9] indicates DNA size in deletions shown in lanes 7-10 are all slightly different despite their inadequate resolution in the FIGE (Figure 3A). This is indicated in the schematic diagram in panel $\mathrm{C}$ of Figure 3 , and the actual sequences included in [Additional File 1].

Progressive end-deletions using the lox66 transposons were generated at an overall efficiency of $10-20 \%$ of that obtained with the wild type loxP transposons, depending on whether other recombinogenic sites existed in the genomic DNA insert of the BAC clone. For example, efficiency was at the lower end of $\sim 10 \%$ with BAC clone $\mathrm{C}$; which consistently generates a large percentage of lox-Cre independent internal deletions. As much as $\sim 40 \%$ of clones isolated comprise internal deletions of that particular size shown in lane 6, Figure 3A, when a wild type loxPmarkerless transposon is used. Note that lox-Cre independent internal deletions of genomic insert are readily isolated here because there is no selection for transposition itself in these markerless transposons, only selection for deleting insert DNA to less than P1 headful packaging capacity (see [20] for discussion). 


\section{FIGE of lox66 substituted BAC deletions}

A

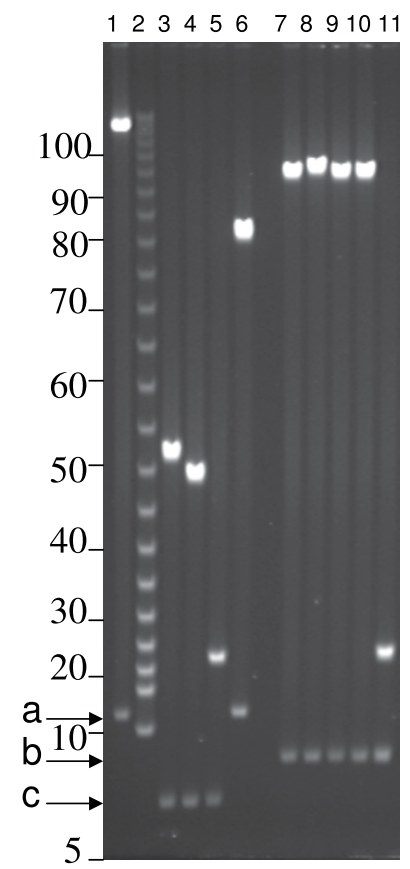

B

PTARBAC2.1 vector

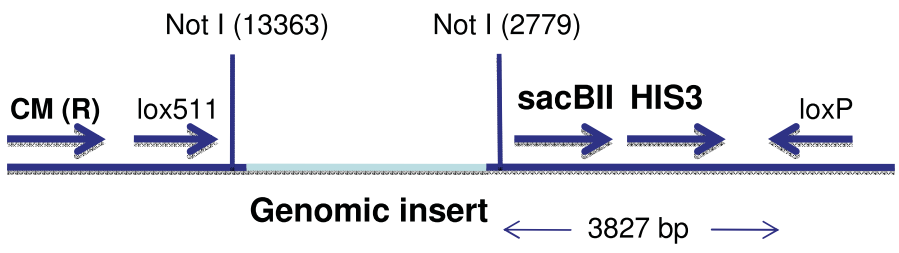

Not I-Not I vector band is $10584 \mathrm{bp}$

\section{Ends of deletion clone in lanes 7-10}

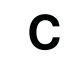

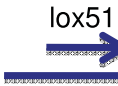

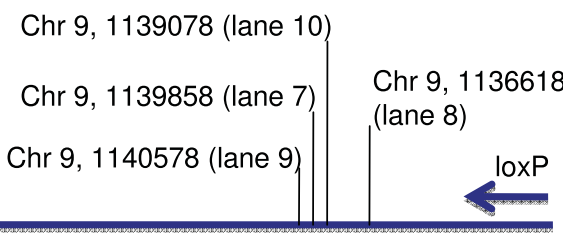

BAC-D

Figure 3 FIGE analysis of BAC DNA isolated from deletions generated with lox66 transposons: Panel A: The BAC DNA was digested with Not I prior to FIGE. Lane 1 shows DNA from starting BAC-C. Lanes 3-5 and 7-11 display DNA from BAC deletions generated by Crerecombination of lox66 with loxP using transposons pTnLox66(B)markerless and pTnLox66(B)markerless Enhancer-Trap transposon, respectively. Lane 6 shows DNA from a loxP-Cre independent internal deletion. Lane 2 shows a 5 kb ladder. The vector DNA bands generated with Not I a, $\mathbf{b}, \mathbf{c}$, are indicated by the arrows to the left. Size of vector bands $\mathbf{b}$ and $\mathbf{c}$ are consistent with loxP-Cre dependent recombinations, while vector band $\mathbf{a}$ arises from starting BAC-C or from an internal deletion in BAC-C. DNA from BAC deletions made with pTnLox66(B)markerless transposon generated the Not I vector band of the expected size ( 6.6 kb) shown in lanes 3-5 of Figure 3 (marked by arrow c). The BAC deletion shown in lane 6 arises from an internal deletion in the genomic insert DNA, and is independent of lox-Cre recombination. The vector DNA band upon Not I digestion of this clone is $10.6 \mathrm{~kb}$ in size, and is identical to that of starting BAC clone C (displayed in lane 1, Figure $3 \mathrm{~A}$ and marked by arrow a). This vector DNA band serves as a characteristic identifying feature for internal deletions [20], and can comprise 90\% of isolates in end-deletions made with certain BAC clones (PKC unpublished observations). These arise due to recombinogenic sites in insert DNA (discussed in [20]). Panel B: A schematic representation of the Not I sites in pTARBAC2.1 vector DNA in BAC clones is shown. Panel C: Location of ends of lox66 substituted deletion clones in lanes7-10 on zebrafish chromosome 9 is indicated. These were obtained by BLAST analyses of the BAC end sequences derived with Seq 1 primer [Additional File 1] with the zebrafish genome sequence.

End-deletions with the lox66 transposons approached $\sim 20 \%$ with a different BAC clone $\mathrm{D}$, containing $\mathrm{APPb}$ gene sequences from a different region of the chromosome. BAC-D produces far fewer internal deletions (not shown). The lower efficiency of generating end-deletions with either of the two lox66 transposons is in sharp contrast to the transduction experiments with phage P1, displayed in Table 1, where no difference was observed between wild type loxP and lox66 carrying plasmids.

\section{Sequencing newly created end of lox66 BAC deletions in} both directions with transposon based primers

The DNA from BAC deletions generated with the two lox66 transposons was sequenced with primers complementary to the transposon end (see [9] for details).
These are seq 1, and seq 4-compliment for deletions made with pTnLox66(B)markerless transposon, and seq 1 and LF8-compliment for deletions made with pTnLox66(B)markerless Enhancer-Trap transposon. While primers Seq 1 and Seq 4 have been described earlier [9], primer LF8-compliment was designed from within the intron enhancer of Amyloid Precursor Protein $(\mathrm{APPb})$ gene [2]. The results are shown schematically in Figure 4.

The Seq 1 primer sits 38 nucleotides from the transposon end, and sequences generated with it read outward into the genomic insert DNA (9). Sequencing BAC deletions displayed in lanes 3-5 and 7-11 of Figure 3A with Seq 1 primer indicate that progressive truncations have occurred from the loxP end of genomic insert 
DNA in each of these clones from starting BACs $\mathrm{C}$ and $D$ respectively. BLAST analyses of these sequences determine the deletion end points (shown in Figure 3C), and are consistent with their mobility on FIGE.

Sequences of BAC deletions obtained using either Seq 4-compliment or LF8-compliment read in the opposite direction, as indicated in Figure 4. The results clearly demonstrate that the loxP site in the parent BACs have been replaced with lox66. The sequences in Figure 4 also indicate that restriction enzyme sites in the starting transposon have been preserved in the BAC deletions (compare restriction sites in Figures 2 and 4).

\section{Discussion}

We describe a general approach to replace the wild type loxP sequence located at one end of genomic DNA inserts in all public domain BACs with a lox66 site. The procedure uses a lox66 transposon to trim insert DNA from the $\operatorname{lox} P$ end and simultaneously replace the original $\operatorname{lox} P$ with a lox66 site. Replacement of $\operatorname{lox} P$ with lox66 occurs with high fidelity. Although the genomic DNA insert is truncated in the process, the size of insert DNA remaining in the lox66 BAC can be as large as 105 $\mathrm{kb}$, the limit encountered by the $\sim 110 \mathrm{~kb}$ packaging capacity of the P1 phage head. The resulting $105 \mathrm{~kb}$ insert DNA size, the remainder being BAC vector, is unlikely to be a drawback in most applications; because a majority of vertebrate genes can be housed in their entirety within this size limit: more than half of noncoding gene-regulatory sequences in vertebrates are located within this span of DNA adjoining start sites of genes [21]. Therefore lox66 BACs housing entire genes 
in their chromosomal contexts can now be stably integrated to pre-positioned lox71 sites in chromosomes using Cre recombination as described earlier for small plasmids $[4,5]$.

The fidelity of substituting lox66 for loxP in BACs is high: No truncations occurred from the lox511 side of genomic DNA insert in our experiments using the lox66 transposons. This should have been easy to detect because a different sized vector DNA band would have been generated upon Not I digestion of the DNA (see Figure $3 \mathrm{~B}$ ). The high fidelity of recombination observed in the $\operatorname{loxP}$ site substitutions is consistent with the transduction experiments described here (compare rows 1, 2 and 5, 6 with row 3 in Table 1) and with earlier studies using P1 phage-generated Cre protein $[7,16]$.

The results shown in Figures 3 and 4 indicate that additional changes, such as incorporating enhancertraps [2] or other reporter and/or selectable marker genes can readily be made in the lox66-BACs during the substitution process. The methodology should facilitate targeting functionalized lox66-BACs to a pre-positioned lox71 site on the chromosome to generate transgenic animals. The approach should be of special interest in systems, such as zebrafish, where "knock-in" technology using homologous recombination are un-available due to genome duplication in an ancestral teleost [22]. Targeted integration using this strategy has been reported recently in zebrafish; but only to integrate small lox66 plasmids to lox71 sites on chromosomes [23].

Targeting loxP plasmids to vertebrate genomes can be affected both by the DNA topoisomerase activity of Cre protein and cryptic lox sites in chromosomes [24-27]. The topoisomerase activity of Cre is unavoidable, and might explain the low efficiency of integrating small lox66 plasmids to chromosomal lox71 sites in zebrafish [23]. Cryptic lox sites are also likely to reduce efficiency, although integration at those sites is expected to be less efficient than at authentic lox71 sites. Despite these potential complications, targeting of small lox66 plasmids to a lox71 site has been successful in zebrafish [23], plants [4] and mouse ES cells [5,3], and therefore lox66 BACs should be targetable in a similar manner.

We believe using a lox66 transposon to substitute the loxP site in BACs should be easier than using homologous recombination in E.coli because the same lox66 transposon can be used with all BACs in the public domain. Recombineering approaches on the other hand require building at least one arm of homology for each BAC [28-30]. Additional alterations to the BAC are easy to incorporate with lox66 transposons, but difficult otherwise.

The overall efficiency of making end-deletions with the lox66 transposons is 5-10 fold lower compared to loxP transposons, despite there being no difference between the two in phage P1 transduction of plasmids containing either site. This was puzzling. The BAC end- deletion procedure can be broken down into three discrete Cre-mediated recombination steps (shown schematically in Figure 5), namely 1) creating the lox66-loxP deletion in BAC after the lox66 site transposition, 2) generating the co-integrate between deleted lox66-BAC and P1 phage, and 3) circularizing the linear BAC DNA packaged in the phage head upon re-entering fresh bacteria (see references 16, 20 for details). Each of these steps requires Cre recombination between loxP and lox66 sites. While steps 2) and 3) are common to both $\mathrm{P} 1$ transductions and $\mathrm{BAC}$ end-deletion procedures, step 1) is unique to the latter. Note also that steps 1) and 3) most likely occur during the early stages of the P1 infection, with lower levels of Cre protein around, while step 2) occurs late in infection with probably higher levels of Cre protein in the cell. It is tempting to speculate therefore that step 1) is likely to be more stringent than step 2), and might discriminate between loxP and lox66 sites so as to slightly disfavor the loxPlox66 compared to a loxP-loxP recombination. Thus fewer truncations are likely to occur with lox66 inserted into the genomic DNA; resulting in fewer BAC inserts capable of packaging both the lox66 and loxP sites within the same phage head that ultimately allows them to be circularized in the next round of infection $[16,20]$. Such rationalization would also require that a similar discrimination in step 3) is not enough to lower survival of the truncated linear BAC DNA flanked by loxP-lox66 within phage P1 heads through circularization. A delay in degradation of the linear DNA upon entry into the cell might be sufficient to overcome this discrimination. Also other recombinases in the cell might help out in the process of circularization, as noted earlier [16]. Loss of stringency of lox-Cre recombinations with higher levels of Cre protein, proposed for step 2), is most likely responsible for the wide range of promiscuity observed in previous studies where Cre was expressed constitutively in cells or used in vitro [11-15,17].

Several interesting conclusions can be drawn from considering the asymmetry of the lox-Cre recombination reactions involved in the substitution process. There is directionality to the loxP site arising from the 8 -base asymmetric spacer. Cre-recombination of identical spacer lox sites occur readily, mutant or otherwise, while those between non-identical spacers is severely restricted [16,7]. Thus substitution of the lox511 site at the other end of insert DNA in BACs with lox66 is likely feasible when it also carries the same spacer mutation as lox511. The directionality of the lox-Cre recombination should also prevent substitution of $l o x 71$ for loxP in BACs: because the arm mutation in lox71 is on the rear end of the arrow shown in Figure 1 (top 


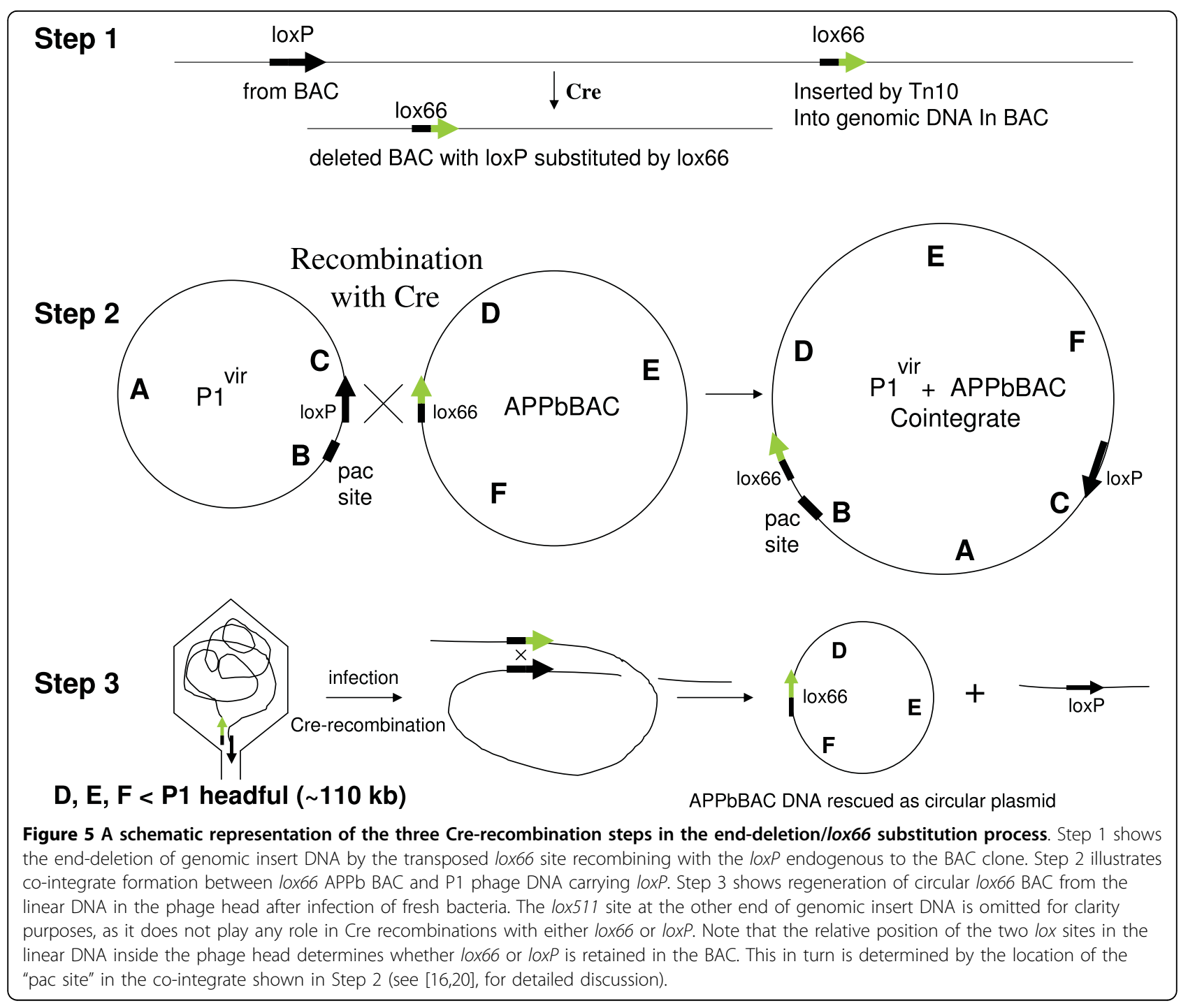

panel), the Cre recombination after transposition of a lox71 site into genomic DNA would result in lox71 ending up in the deleted portion of the genomic insert (see bottom panel of Figure 1). However, transduction of plasmids carrying lox 71 by phage $\mathrm{P} 1$ is not affected by this asymmetry: the lox71 site should end up in segment F-C of cointegrate rather than segment B-D as in the case of lox66 (refer to Step 2 of Figure 5). The linear DNA in the P1 phage head should then be flanked by $\operatorname{loxP}$ at left and lox71 on right, respectively (Step 3 of Figure 5). Upon Cre-recombination this DNA should be able to circularize with lox71 in the BAC and loxP in the small linear piece of DNA from the termini (step 3 Figure 5).

The lower efficiency of end-deletions with lox66 compared to loxP transposons should not pose a hurdle because several thousand deletion clones are generated in each deletion/substitution experiment, and one can screen a sufficient number of clones to obtain the desired number of lox66 substituted BAC deletions. Using this end-deletion/substitution procedure, BACs as large as $110 \mathrm{~kb}$ with loxP replaced by lox66 can be generated with minimal effort using the same lox66 transposon for all BACs in the public domain.

Additional file 1: Sequences of BAC deletion ends. Sequences of the end points of the BAC deletions shown in lanes 7-10, Figure 3, on zebrafish chromosome 9 . These were obtained by direct BAC endsequencing with the Seq 1 primer located in the transposon end retained in the deletion clone.

Click here for file

[http://www.biomedcentral.com/content/supplementary/1756-0500-3-38S1.DOCX]

\section{Abbreviations}

BAC: bacterial artificial chromosome; FIGE: Field inversion gel electrophoresis; APPb: Amyloid Precursor Protein. 


\section{Acknowledgements}

We thank Dr. Faye Calhoun, Cheryl Harrington, Rosalind Grays, Connie Key and Kara Battle, for support and encouragement. This work was supported in part by MBRS-SCORE grant \# SO 608049 from the NIGMS, and EXPORT grant \#1P20 MD00175-01 from the NIH and funds from the North Carolina Biotechnology Center.

\section{Author details}

'Department of Chemistry, North Carolina Central University, 1801 Fayetteville Street, Durham, NC 27707, USA. Julius L Chambers Biomedical/ Biotechnology Research Institute, North Carolina Central University, 1801 Fayetteville Street, Durham, NC 27707, USA. ${ }^{3}$ Department of Biology, North Carolina Central University, 1801 Fayetteville Street, Durham, NC 27707, USA.

\section{Authors' contributions}

PKC carried out the design of experiments, lox66 transposon retrofitting of BACs, analyses of data and writing of manuscript. LAS, NS, VLR and TLM screened BAC deletion libraries using FIGE, end-sequencing of lox66 substituted BACs and bio-informatic analyses of data. KRH helped with writing, provided critical evaluation of scientific content of manuscript and helped with funds. All authors read and approved the final manuscript.

\section{Competing interests}

The authors declare that they have no competing interests.

Received: 7 October 2009

Accepted: 19 February 2010 Published: 19 February 2010

\section{References}

1. Bronson S, Plaehn E, Kluckman K, Hagaman J, Maeda N, Smithies O: Singlecopy transgenic mice with chosen-site integration. Proc Natl Acad Sci USA 1996, 93:9067-9072.

2. Shakes LA, Malcolm TL, Allen KL, De S, Harewood KR, Chatterjee PK: Context dependent function of APPb Enhancer identified using Enhancer Trap-containing BACs as Transgenes in Zebrafish. Nucleic Acids Research 2008, 36:6237-6248.

3. Sauer B, Hendenson N: Targeted insertion of exogenous DNA into the eukaryotic genome by the Cre recombinase. New Biol 1990, 441-449.

4. Albert $H$, Dale EC, Lee E, Ow DW: Site-specific integration of DNA into wild-type and mutant lox sites placed in the plant genome. Plant J 1995, 7:649-659.

5. Araki K, Araki M, Yamamura K: Targeted integration of DNA using mutant lox sites in embryonic stem cells. Nucleic Acids Res 1997, 25:868-872.

6. Chatterjee PK: Retrofitting BACs and PACs with LoxP Transposons to Generate Nested Deletions. "Bacterial Artificial Chromosomes". Methods in Mol Biology 2004, 1:231-241.

7. Shakes LA, Garland DM, Srivastava DK, Harewood KR, Chatterjee PK: Minimal Cross-recombination between wild type and lox511 sites in vivo facilitates Truncating Both Ends of Large DNA Inserts in pBACe3.6 and Related Vectors. Nucleic Acids Research 2005, 33:e118.

8. Chatterjee PK, Baker JC Jr: Preparing Nested Deletions Template DNA for Field Inversion Gel Electrophoresis Analyses and Position-Specific End Sequencing With Transposon Primers. "Bacterial Artificial Chromosomes" vol 1, pp 243-254. Methods in Mol Biology series The Humana Press IncShaying Zhao, Marvin Stodolsky 2004, 255.

9. Chatterjee PK, Yarnall DP, Haneline SA, Godlevski MM, Thornber SJ, Robinson PS, Davies HE, White NJ, Riley JH, Shepherd NS: Direct Sequencing of Bacterial and P1 Artificial Chromosome Nested-deletions for Identifying Position-Specific Single Nucleotide Polymorphisms. Proc Natl Acad Sci (USA) 1999, 96:13276-13281.

10. Hoess RH, Wierzbicki A, Abremski K: The role of the loxP spacer region in P1 site-specific recombination. Nucleic Acids Res 1986, 14:2287-2300.

11. Lee G, Saito I: Role of nucleotide sequences of loxP spacer region in Cremediated recombination. Gene 1998, 216:55-65.

12. Siegel RW, Jain R, Bradbury A: Using an in vivo phagemid system to identify non-compatible loxP sequences. FEBS lett 2001, 505:467-473.

13. Langer SJ, Ghafoori AP, Byrd M, Leinwand L: A genetic screen identifies novel non-compatible loxP sites. Nucleic Acids Res 2002, 30:3067-3077.

14. Missirlis $\mathrm{PI}$, Smailus DE, Holt RA: A high-throughput screen identifying sequence and promiscuity characteristics of the lox $\mathrm{P}$ spacer region in Cre-mediated recombination. BMC Genomics 2006, 7:73-85.
15. Sheren J, Langer SJ, Leinwand LA: A randomized library approach to identifying functional lox site domains for the Cre recombinase. Nucleic Acids Res 2007, 35:5464-5473.

16. Chatterjee PK, Shakes LA, Srivastava DK, Garland DM, Harewood KR, Moore KJ, Coren JS: Mutually exclusive recombination of wild-type and mutant loxP sites in vivo facilitates transposon-mediated deletions from both ends of genomic DNA in PACs. Nucleic Acids Res 2004, 32:5668-5676.

17. Wang Z, Engler P, Longacre A, Storb U: An efficient method for highfidelity BAC/PAC retrofitting with a selectable marker for mammalian cell transfection. Genome Res 2001, 11:137-142.

18. Hartung M, Kisters-Woike B: Cre mutants with altered DNA binding properties. J Biol Chem 1998, 273:22884-22891.

19. Guo F, Gopaul DN, Van Duyne GD: Structure of Cre recombinase complexed with DNA in a site-specific recombination synapse. Nature 1997, 389:40-46.

20. Chatterjee PK, Mukherjee S, Shakes LA, Wilson W III, Harewood KR, Byrd G: Selecting Transpositions of a Markerless Transposon Using Phage P1 Headful Packaging: New Transposons for Functionally Mapping Long Range Regulatory Sequences in BACs. Analytical Biochemistry 2004, 335:305-315.

21. Woolfe A, Goodson M, Goode DK, Snell P, McEwen GK, Vavouri T, Smith SF, North P, Callaway H, Kelly K, Walter K, Abnizova I, Gilks W, Edwards YJ, Cooke JE, Elgar G: Highly conserved non-coding sequences are associated with vertebrate development. PLoS Biol 2005, 3:e7.

22. Kleinjan DA, Bancewicz RM, Gautier P, Dahm R, Schonthaler HB, Damante G, Seawright A, Hever AM, Yeyati PL, van Heyningen V, Coutinho P: Subfunctionalization of duplicated zebrafish pax6 genes by cisregulatory divergence. PLoS Genet 2008, 4:e29.

23. Liu WY, Wang Y, Qin Y, Wang YP, Zhu ZY: Site-Directed Gene Integration in Transgenic Zebrafish Mediated by Cre Recombinase Using a Combination of Mutant Lox Sites. Marine Biotechnology 2007, 9:420-428.

24. Abremski K, Wierzbicki A, Frommer B, Hoess RH: Bacteriophage P1 CreloxP site-specific recombination. Site-specific DNA topoisomerase activity of the Cre recombination protein. J Biol Chem 1986, 261:391-396

25. Thyagarajan B, Guimaraes MJ, Groth AC, Calos MP: Mammalian genomes contain active recombinase recognition sites. Gene 2000, 244:47-54.

26. Schmidt EE, Taylor DS, Prigge JR, Barnett S, Capecchi MR: Illegitimate Credependent chromosome rearrangements in transgenic mouse spermatids. Proc Natl Acad Sci USA 2000, 97:13702-13707.

27. Semprini S, Troup TJ, Kotelevtseva N, King K, Davis JR, Mullins LJ, Chapman KE, Dunbar DR, Mullins JJ: Cryptic loxP sites in mammalian genomes: genome-wide distribution and relevance for the efficiency of BAC/PAC recombineering techniques. Nucleic Acids Res 2007, 35:1402-1410.

28. Gong S, Yang XW, Li C, Heintz N: Highly efficient modification of bacterial artificial chromosomes (BACs) using novel shuttle vectors containing the R6Kgamma origin of replication. Genome Res 2002, 12:1992-1998.

29. Muyrers JP, Zhang Y, Testa G, Stewart AF: Rapid modification of Bacterial Artificial Chromosomes by ET recombination. Nucleic Acids Res 1999, 27:1555-1557.

30. Jessen JR, Meng A, McFarlane RJ, Paw BH, Zon LI, Smith GR, Lin S: Modification of bacterial artificial chromosomes through chi-stimulated homologous recombination and its application in zebrafish transgenesis. Proc Natl Acad Sci USA 1998, 95:5121-5126.

doi:10.1186/1756-0500-3-38

Cite this article as: Chatterjee et al:: Replacing the wild type loxP site in BACs from the public domain with lox66 using a lox66 transposon. BMC Research Notes 2010 3:38. 